\title{
Corrections
}

\section{ENVIRONMENTAL SCIENCES}

Correction for "Variations in atmospheric $\mathrm{CO}_{2}$ growth rates coupled with tropical temperature," by Weile Wang, Philippe Ciais, Ramakrishna R. Nemani, Josep G. Canadell, Shilong Piao, Stephen Sitch, Michael A. White, Hirofumi Hashimoto, Cristina Milesi, and Ranga B. Myneni, which appeared in issue 32, August 6, 2013, of Proc Natl Acad Sci USA (110:13061-13066; first published July 24, 2013; 10.1073/pnas.1219683110).

The authors note that on page 13064, right column, first full paragraph, lines 4-7, "Some of the climatic events linked to such concerns are the severe droughts experienced by the Amazon basin in 2005 and 2010, which reportedly reduced tropical forest NPP by 1.6 to $2.2 \mathrm{PgC} / \mathrm{y}$ and increased tree mortality $(50,51)$ " should instead appear as "Some of the climatic events linked to such concerns are the severe droughts experienced by the Amazon basin in 2005 and 2010, which reportedly reduced tropical forest NPP and increased tree mortality by a total biomass carbon loss of 1.6 to $2.2 \mathrm{PgC}(50,51)$."

On page 13064, right column, first full paragraph, lines 12-17, "Assuming that the large reductions of tropical NPP reported previously $(50,51)$ are realistic, the absence of marked variations of the global $\mathrm{CO}_{2}$ growth rate after the 2005 and 2010 Amazon droughts may imply that the drought conditions also coincidently reduced tropical $\mathrm{Rh}$ along with NPP, resulting in (relatively) small NEE anomalies" should instead appear as "Assuming that the large reductions of tropical forest biomass reported previously $(50,51)$ are realistic, the absence of marked variations of the global $\mathrm{CO}_{2}$ growth rate after the 2005 and 2010 Amazon droughts may imply that the drought conditions also coincidently reduced tropical $\mathrm{Rh}$ along with NPP, resulting in (relatively) small NEE anomalies."

These changes do not affect the conclusions of the article.

www.pnas.org/cgi/doi/10.1073/pnas.1314920110

\section{ECOLOGY}

Correction for "Several scales of biodiversity affect ecosystem multifunctionality," by Jae R. Pasari, Taal Levi, Erika S. Zavaleta, and David Tilman, which appeared in issue 25, June 18, 2013, of Proc Natl Acad Sci USA (110:10219-10222; first published June 3, 2013; 10.1073/pnas.1220333110).

The authors note the following statement should be added to the Acknowledgments: "Data used in this work were supported by the National Science Foundation (NSF) Long-Term Ecological Research (LTER) Division of Environmental Biology (DEB) 9411972 (1994-2000), DEB 0080382 (2000-2006), and DEB 0620652 (2006-2012)."

www.pnas.org/cgi/doi/10.1073/pnas.1314558110

\section{MICROBIOLOGY}

Correction for "CCAAT/enhancer-binding protein $\delta$ facilitates bacterial dissemination during pneumococcal pneumonia in a platelet-activating factor receptor-dependent manner," by JanWillem Duitman, Marcel Schouten, Angelique P. Groot, Joost B. Daalhuisen, Sandrine Florquin, Tom van der Poll, and C. Arnold Spek, which appeared in issue 23, June 5, 2012, of Proc Natl Acad Sci USA (109:9113-9118; first published May 21, 2012; 10.1073/pnas.1202641109).

The authors note that Keren S. Borensztajn should be added to the author list between Angelique P. Groot and Joost B. Daalhuisen. Keren S. Borensztajn should be credited with contributing new reagents/analytic tools. The corrected author line, affiliation line, and author contributions appear below. The online version has been corrected.

\section{JanWillem Duitman ${ }^{a, b}$, Marcel Schouten ${ }^{a, b}$, Angelique P. Groot ${ }^{a}$, Keren S. Borensztajn ${ }^{a}$, Joost B. Daalhuisen ${ }^{a}$, Sandrine Florquinc, Tom van der Poll ${ }^{a, b}$, and C. Arnold Spek ${ }^{a}$}

${ }^{a}$ Center for Experimental and Molecular Medicine, ${ }^{b}$ Center for Infection and Immunity Amsterdam, and 'Department of Pathology, Academic Medical Center, 1105 AZ, Amsterdam, The Netherlands

Author contributions: J.D. and C.A.S designed research; J.D., M.S., A.P.G., J.B.D., and C.A.S. performed research; K.S.B. contributed new reagents/ analytic tools; J.D., M.S., S.F., T.v.d.P., and C.A.S. analyzed data; and J.D., S.F., T.v.d.P., and C.A.S. wrote the paper.

www.pnas.org/cgi/doi/10.1073/pnas.1315309110

\section{BIOCHEMISTRY}

Correction for "SlmA forms a higher-order structure on DNA that inhibits cytokinetic Z-ring formation over the nucleoid," by Nam K. Tonthat, Sara L. Milam, Nagababu Chinnam, Travis Whitfill, William Margolin, and Maria A. Schumacher, which appeared in issue 26, June 25, 2013 of Proc Natl Acad Sci USA (110:10586-10591; first published June 10, 2013; 10.1073/ pnas.1221036110).

The authors note that the following statement should be added to the Acknowledgments: "SAXS data were collected at the SIBYLS beamline (ALS 12.3.1) funded by Integrated Diffraction Analysis Technologies and Macromolecular Insights on Nucleic Acids Optimized by Scattering (R01GM105404)."

www.pnas.org/cgi/doi/10.1073/pnas.1314545110 